\title{
Application of artificial intelligence in human resource management in the agricultural sector
}

\author{
Svetlana Evseeva ${ }^{1, *}$, Oksana Evseeva ${ }^{1}$, Andrei Burmistrov ${ }^{1}$, and Maria Siniavina ${ }^{1}$ \\ ${ }^{1}$ Peter the Great St. Petersburg Polytechnic University, 29, Polytechnicheskaya, 195251, Saint \\ Petersburg, Russia
}

\begin{abstract}
Digitalization of all spheres of life has become one of the main directions of development in many countries of the world. Artificial intelligence technology has a wide range of applications in various fields. The study aims to identify artificial intelligence usage in human resource management by analysing functions. Qualitative methods are used in the study. As the result, artificial intelligence tools were analysed by matching them on human resource management functions: recruitment, onboarding, assessment, training and development, talent management, salary system, culture and engagement management, management and leadership, misconduct and compliance. Artificial intelligence technologies provide many opportunities for modern companies. Study in detail companies using $\mathrm{AI}$ in $\mathrm{HR}$ functions and possible changes in the HR manager profession and role of HR in business are future research directions.
\end{abstract}

\section{Introduction}

Digitalization affects all spheres of modern society. Digital technologies change not only individual processes, but also entire objects. There were such concepts as digital factories, digital economy, digital competencies, etc [1]. Technology has changed HR processes in terms of collection, storage, usage, and dissemination of information about applicants and employees [2-4]. The European Commission emphasizes artificial intelligence (AI) as a strategically important area and a key driver of economic development. [5]

Investment in AI is growing rapidly. McKinsey [6] estimates that worldwide, tech giants spent $\$ 20$ billion to $\$ 30$ billion on $\mathrm{AI}$ in 2016, of which $90 \%$ was spent on R\&D and deployment, and $10 \%$ on AI acquisition. Venture and private equity funding, grants, and seed investments also grew rapidly, albeit from a small base, to a total of $\$ 6$ billion to $\$ 9$ billion. They have invested more in machine learning. During the pandemic, McKinsey conducted the online survey June, 9-19, 2020, and garnered responses from more than 2000 participants. It also conducted interviews with executives between May and August 2020. On the results of the study, half of the respondents say their organizations have adopted AI in at least one function. While the latest findings show no increase in AI adoption, some companies are capturing value from $\mathrm{AI}$ at the enterprise level, and many are generating

\footnotetext{
* Corresponding author: evseeva_sa@spbstu.ru
} 
revenue and cost reductions at least at the function level. Despite the COVID-19 effect on the economy, the most value from $\mathrm{AI}$ is doubling down on the technology. [7]

Currently, artificial intelligence is being investigated by some scientists from different angles [8]. It provides opportunities that can be used in many areas. In human resource management (HRM), artificial intelligence also has a wide range of uses [9]. Artificial intelligence is important for the connection between education systems and labor markets. Digital technologies help talents find opportunities in the labor market. According to the MGI study, by 2025, online talent platforms can enable 60 million people to find jobs that better match their skills or preferences, and reduce the cost of human resource management, including recruitment, by as much as $7 \%$ [6]. There is growing attention to the problem of lifelong learning. Artificial intelligence capabilities in this area have already begun to attract new players. However, due to the constant development of technology, there is a lack of knowledge about the existing usability of AI in HR management.

Delloitte notes in its Global Human Capital Trends 2020 study that despite the predictions of replacing people with machines in the modern world, there is a search for strategies to integrate AI into teams to create value. And they called it superteams. But 2 gaps hinder this: mainly companies use AI to ensure the quality and productivity of work, and to a lesser extent to assist workers in developing insights; about $17 \%$ of respondents said that their organizations invest in reskilling to support AI strategy. [10] We aimed to identify artificial intelligence usage in human resource management by analysing functions.

\section{Methods}

First of all, it is necessary to define the concept of Artificial Intelligence. The European Commission defines AI as technologies capable of analysing their environment and taking autonomous actions respecting pre-defined values [5]. Another approach in defining AI is based on the ability to learn [11]. However, it excludes more (symbolic) rule-based systems, for example, systems that require predictable and understandable behaviour. It would exclude many traditional AI systems in natural language translation, expert systems, and other types of rule-based reasoning systems would also be excluded. By AI, we mean the ability of a system to correctly interpret external data, learn from it, and use the knowledge gained to achieve specific goals through flexible adaptation [12]. Based on three types of competencies, Andreas Kaplan and Michael Haenlein classify AI systems into three groups: analytical, human-inspired, and humanized AI [12].

According to The Future of Jobs Report 2020 and 2018 companies accelerated the adoption of new technologies. Figure 1 presents AI technology that companies likely to adopt by 2022 and 2025. Russia is in line with global trends in this area, but we see that the situation has deteriorated slightly. Perhaps one of the reasons is the COVID-19 pandemic. 


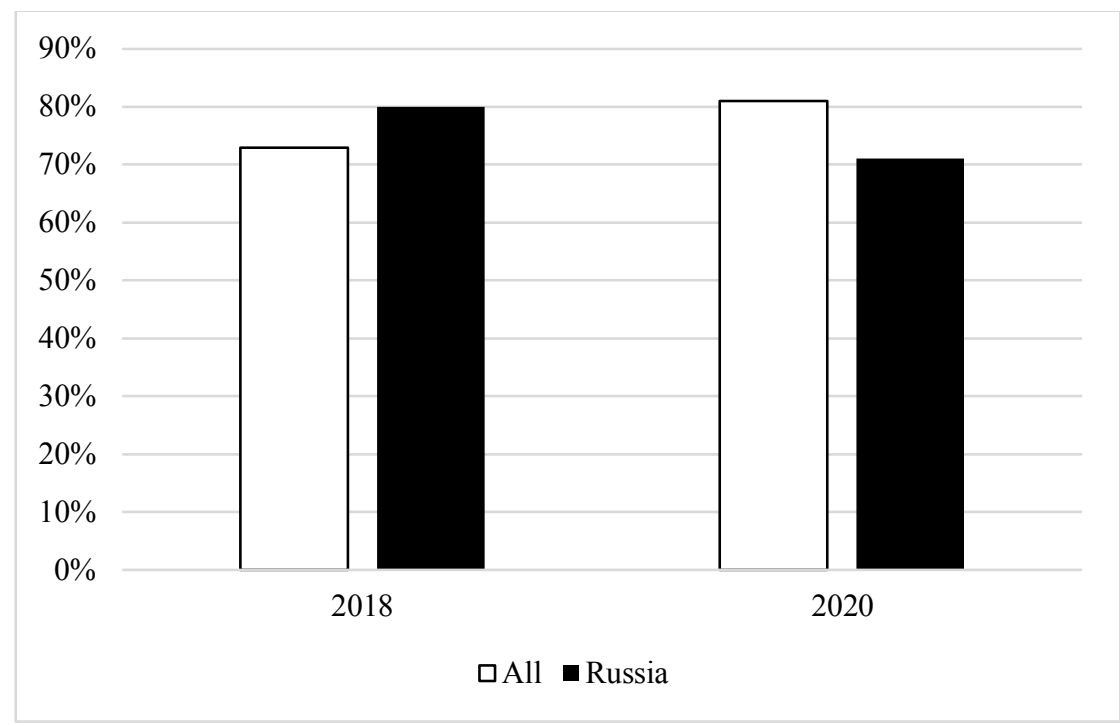

Fig. 1. AI by proportion of companies likely to adopt it (based on The Future of Jobs Report 2020 and 2018).

We conducted a qualitative analysis, focusing on products and services that may be provided to companies based on AI. Open sources data were used. The data were collected from scientific articles, consulting companies' reports, official websites of companies and sites that aggregate information about various products using artificial intelligence. Data collection was carried out on the main functions of HRM. As a result, the use of AI spanned recruitment, onboarding, assessment, training and development, compensation and benefits, culture and engagement management, misconduct and compliance. We analyzed international and Russian experience.

Currently, there is little empirical data on this topic, despite its relevance and rapid development. Therefore, it was important to collect the available data and draw conclusions based on this about the directions of further research.

\section{Results}

Artificial intelligence is used to perform many functions of HRM (Fig. 2). AI systems using statistical data, can "predict" and "learn" by forming solutions and optimizing them based on criteria. 


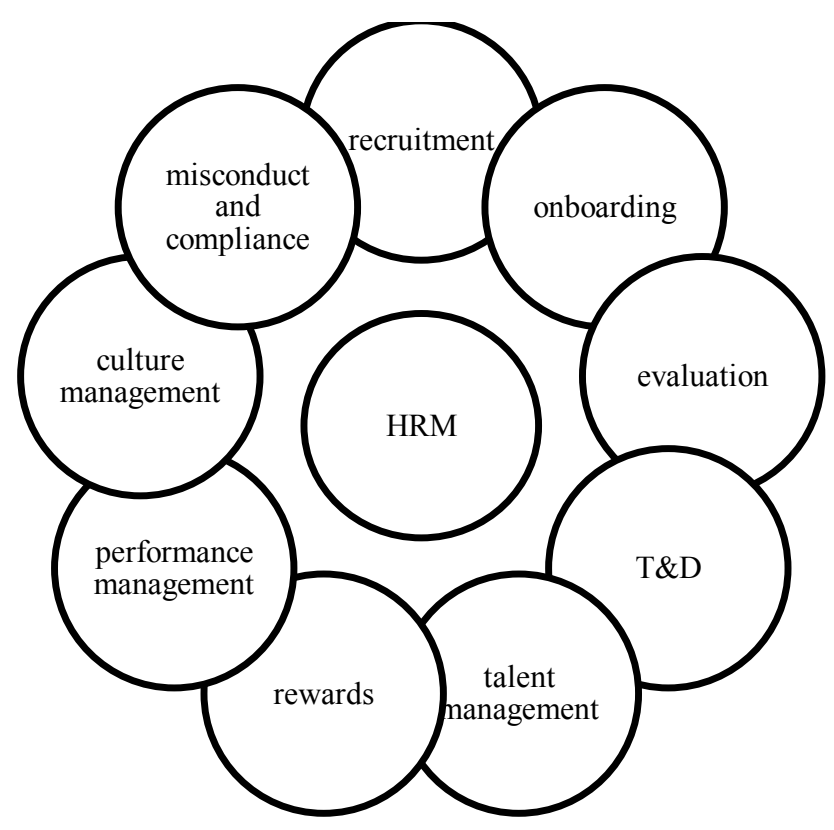

Fig. 2. AI application in HRM functions.

In recruiting, using artificial intelligence is already quite wide. The AI system uses all demographics, work experience, and interview questions for candidates, and then predict the effectiveness of doing their job. Examples of such companies in foreign markets are HiredScore, Pymetrics, HireVue, IBM and other companies. AI helps you write the right text to attract candidates to the company (for example, Textio). Many startups (Blendoor, Ideal, Harver, HiringSolved, Headstart) are looking for "passive" candidates in social networks and professional communities who can be effective in certain positions. [13] Linkedin's artificial intelligence gives candidates feedback on their interview responses.

Modern robots based on artificial intelligence are used in the mass selection and robots can track expert profiles even on social networks and make lists of those who are suitable for the company. Some programs still ask clarifying questions to selected people - via Facebook's message service and other instant messengers, and then broadcast the response to the HR manager. For example, the robot FirstJob - Californian job search platform for beginners.

Russia also has technologies and companies that use them. One of the actively developing areas is the use of robots. Examples are robot Vera and Yermil. Robots are connected to the primary personnel selection, capable of speaking and recognizing speech. They look for resumes on recruiting sites according to the given parameters, calls potential applicants to the specified phones and tells them about the company's vacancies. If the candidate is interested, the robot informs the HR department.

AI-based chatbots can conduct a dialogue with a candidate and then assess its potential effectiveness. It is important that chatbots with artificial intelligence, unlike conventional ones, do not require direct programming of rules: how to answer human questions. Moreover, artificial intelligence can interpret even an indirect response from the applicant. Abroad, chatbots are developed by the companies Mya Systems, Paradox, Text Recruit. In Russia - Robot Vera and Yandex.Talents.

Using AI to analyze video interviews helps to make decisions about the selection of candidates. Based on the existing base of video interviews, it is possible to recognize 
speech, voice, text complexity, facial expressions, and then compare it with data on each person about how successfully he passed intellectual tests, a potential assessment test, a complex assessment procedure. From the data obtained, it is possible to identify in the initial videos those patterns that correspond to successful or unsuccessful candidates and to draw up a complex model showing how much a given person can pass complex assessment tools. A three-minute video is enough for this. Thus, it is possible to conduct hundreds of thousands of video interviews, analyze them using artificial intelligence, and predict the performance of people with great accuracy. An example is Interviewer.Ai abroad and Ecopsy in Russia.

Universal platforms based on artificial intelligence helps to combine all functions. An example of such a platform in the Russian market is Skillaz, an automated cloud-based recruiting platform based on artificial intelligence. The service provides a full range of tools at all stages of recruitment - automatic search and preliminary assessment of candidates, video interview recording, security checks, bidding, Skillas is already actively used by many large Russian companies - Sberbank, Megafon, KPMG, Gazprom Neft [14]].

The work in the field of onboarding new employees can be distinguished close to the topic of recruiting. Artificial intelligence allows customization of the process to cater to individual employees. Algorithms can be integrated within the software for:

- $\quad$ explaining job profile, duties, and benefits;

- communication within the company;

- $\quad$ answering questions by new employees;

- document verifications;

- device requests, and more.

Chatbots in this area serve as a more "human" knowledge base. An employee who has just arrived can ask the bot about the structure of the company, schedule, benefits and other questions that are important in the first months of work. An example of such a startup is Leena.ai.

AI is also used to assess personnel. Almost all gamified assessment is now based on artificial intelligence. A participant performs a large number of actions in one game session in puzzles, arcades, strategies, which can be used to predict his effectiveness in work. The analysis of such unstructured information comes with machine learning technologies. In 2012-2015, the US experienced a boom in gamified valuation startups (Pymetrics, Arctic Shores, Scoutable, Knack). In Russia, gamification using artificial intelligence is still not widespread.

Delta.ai tool - specially shaped questionnaires using AI. Essentially, employee appraisals are done for internal recruiting purposes. To begin with, colleagues and a leader are asked how this or that person actually behaves, what are his strengths and weaknesses. These cross-sectional surveys are conducted for many employees. Then the assessed passes a specially designed questionnaire. As a result, the machine analyzes the intersections of responses and actual behavior, identifying patterns. It turned out that the patterns are not at all obvious, which means that it is almost impossible to guess them.

In Russia, Ecopsy has a tool that evaluates employees based on AI - a competency model based on HR analytics. The quality of what underlies the assessment largely determines its result and benefit. By analyzing data using artificial intelligence, you can find the behavior that is related to the performance in a particular company and form a truly working competency model.

In training and development, two classes of artificial intelligence technologies can be distinguished. The first analyzes the performance of the employee, feedback from colleagues, test results and build individual development programs. After a while, 
performance measurements and a survey of colleagues are repeated, and the development program is adjusted.

The second class of technologies helps to build skills in employees that will increase their effectiveness. Usually, the list of skills is taken from the development program. Every day, a notification is sent to the employee's phone with a list of actions that he must perform today. At the end of the day, the employee confirms what actions he has taken, and the next day he receives a new list.

Traditionally, Kirkpatrick's model [1] is used to assess the effectiveness of training, and it is believed that at each subsequent level it is more difficult to assess the effectiveness of training. However, with the advent of artificial intelligence, assessing the latter level is an order of magnitude easier than any other. An important condition is a sufficient number of people - tens, and preferably hundreds and thousands, to identify patterns easier and more accurate.

The performance of an employee can be influenced by many factors: work experience, age, gender, assessment of competencies, including learning. Learning is just one factor. Filling all the data into the model and comparing the performance of trained and untrained people is fairly straightforward. Artificial intelligence can isolate the impact of learning (or even a separate module, which is especially important with the current trend towards microlearning) on efficiency.

Algorithms can track and learn the knowledge, behavior, and actions of the most effective team members and then simply explain what is needed to fit them. Similar algorithms are used in the field of educational platforms (Degreed, EdCast, Filtered, Volley, Clustree, Workday, etc.)

In Russia, the case of using the Robot Albert for training is interesting. The training goes through messengers and simulators with artificial intelligence and does not require the installation of special software. You can create a course of almost any complexity and duration without programming skills in a simple and powerful built-in bot editor. Albert can be configured to microlearning in groups and individually. To reinforce the skills, Albert organizes training in the form of calls and/or simple audio simulators, and provides the participant with detailed feedback. Customizable gamification and Knowledge Management System available. Albert translates "into figures" every action or inaction of the participant and generously shares statistics and insights both with students and with their leaders.

AI-powered talent management is similar to learning. We can isolate different factors and understand what is a factor of success in the company and promotes career advancement. Also, during the analysis, we can understand which assessment tools work best for promotion in a particular organization. The approach is becoming a thing of the past: to copy the behavior of sample employees according to some criterion and look for the same ones for promotion.

For example, there is a social intelligence test that uses multiple-choice cases. There are answers from top managers and line managers. Further, the answers of new survey participants are compared by the artificial intelligence system with the constructed model and identify those who respond as top managers. Most of the answers by themselves do not mean anything, they do not work without context, but begin to mean only when a person has answered other questions in a certain way, because relationships are important. It is artificial intelligence that can quickly build these complex chains.

The results of employee surveys can be used to identify potential opinion leaders who can be promoted in the organization.

Artificial intelligence systems are able to understand what pattern exists in the organization of the salary system and formalize it. A huge array of information is collected 
from various sources (assessments of the head; mutual assessment of positions by employees in terms of importance, complexity, cost of error; data on education, professional certification, retraining, completed training; information on the performance of employees and much more) a huge array of information is collected, which is processed by artificial intelligence and is into a fairly clear and understandable system of remuneration. It is important to note that the system also takes into account the department where the employee works, and hence the "complexity" of the labor market for him, the region of work of employees, again due to the nuances of the personnel situation.

AI systems can also be used in culture management and employee engagement. In terms of employee well-being and employee engagement, AI is used to identify behavioral patterns that affect performance degradation. New analytic tools can identify signs of stress and alert HR or line management. AI-enabled systems will take over the task of observing and analyzing employees' mood before and after a client call. The HR can then decide if the individual needs a break or can continue. Limeaid, VirginPulse, Glint, Ultimate Software, Peakon and others can be distinguished. Artificial intelligence help managers understand employees happiness. So, the Veriato algorithm analyzes the text of emails, chat messages and signal, for example, a sharp change in tone from positive to negative.

Artificial intelligence systems need richer data to perform qualitative data analysis. To do this, you need to ask more open-ended questions when analyzing engagement. After that, modern artificial intelligence systems can interpret the answers using text analysis based on mathematical modelling. A variation of this technology - artificial intelligence can analyze correspondence in the mail and corporate messengers and highlight the least engaged and satisfied employees.

With the help of artificial intelligence, it is possible to study the formation of teams and study how closed the department is: what roles people play to each other, what roles they want to perform and whatnot, what relationships exist between employees. In an organization of ten thousand people, one such study yields hundreds of thousands of data points, so artificial intelligence is needed to interpret this volume of data. An example of a company that can provide these capabilities is Ecopsy.

AI can be used to predict the probability of an employee being fired. However, one of the problems of using the results of the analysis - it is not clear what to do with them - we can identify people at risk, but we cannot understand why they might quit. Some systems help predict the quit of a valued employee and offer timely promotions or other incentives to retain them. Entelo, IBM, Workday and Microsoft have such programs. For example, the Workday application uses a database of 100,000 employees from different companies for 25 years and calculates the individual exit risk using 60 parameters. In Russia prediction of layoffs is used by Sberbank for the time being only for consultants.

In management and leadership, there are AI-powered learning tools and systems that solicit feedback, read comments, and guess the mood of employees and teams. They use this data to correlate personal and team performance with those of higher-performing teams, allowing managers and supervisors to understand what is needed to get the job done more productively. Examples are Reflektiv, BetterWorks, Ultimate Software, Zugata, Humanyze, etc.

There are also great opportunities in the area of misconduct and compliance. AI can view organizational network data (emails, comments) and identify stress areas, potential ethics violations, and many other forms of non-compliance risk, as well as monitor compliance so that managers can intervene to prevent fraud. Example TrustSphere, Keencorp, Volley, Cornerstone, etc. In the field of security, AI is able to identify behavioral factors that lead to accidents. 
The technologies track employees' activities in the workplace. For example, the Veriato program takes screenshots at regular intervals and sends information about sending messages to the Veriato Server. There, artificial intelligence analyzes them and looks for anomalies indicating low employee productivity, attempts to unauthorized access to classified information or the intention to leave the company.

Also, it is possible to track the movement of employees around the office. Bluvision has developed radio badges that transmit information about the movement on the territory to which access is restricted and send a warning. Using the same system, it is possible to find out how much time an employee spends at his desk, and how much time in a buffet or restroom.

During the pandemic, the Landing AI Startup developed a system for monitoring social distancing at work. The trained neural network monitors employees by video calculate the distance between them and issue a warning if the distance is less than the allowable one. The tool is intended for use in working environments such as warehouses and workshops. The technology was developed in response to customer requests such as Foxconn. The detector can be easily integrated into existing video surveillance systems. An alarm can be selected as a violation notification.

\section{Discussion}

We found that companies use the capabilities of artificial intelligence in the following HRM functions: recruitment, onboarding, assessment, training and development, talent management, salary system, culture and engagement management, management and leadership, misconduct and compliance. Most of the functions are covered by AI, but very unevenly.

As noted, [15] in practice, HR often collects a diverse array of big data with unknown value to predict and explain the results. It should be used to describe, classify, and predict various phenomena and results of interest to organizations.

Important advantages of artificial intelligence systems are the objectivity of decisions and the high processing speed of big data. IBM conducted a study in 2017 on how cognitive computing will influence HR. The research revealed that artificial intelligence can help professionals make quick decisions on day-to-day matters [2]. When we teach the system, we also pass on some of our biases to it, but since the system learns from the biases of many people, the likelihood of actual subjectivity is very small. Another important feature is the ability to quickly compare facts and identify relationships due to the high speed of information processing.

Marwan Mohamed Abdeldayem and Saeed Hameed Aldulaimi agree that AI plays a great role because it impacts facilities and their operations significantly, and workers must be fully prepared to support digital transformation, otherwise human resources will be at risk. Frey and Osborne [16] imply that advances in technology will lead to a reallocation of low-skill workers to tasks that are non-susceptible to computerization. In this situation, creative and social skills will be required of them. The increased use of information technology within an organization has radically changed the types of businesses and skills required for certain activities such as training, development and organization. Therefore, the human resources department must have basic information generated from computer services to overcome the problem that most large organizations currently face and increase its ability to provide valuable information to management for making decisions. The usage of AI in HR functions can help reduce the time on administrative tasks, reduce the burden on shared service centers and help desks by performing HR operations and responding to routine inquiries, recruiting and retaining staff. HR professionals will be able to focus more 
on strategic planning at the organizational level. [17]

However, the use of artificial intelligence in HR management is constrained by several factors. When hiring staff, some feature values are missing or unreliable although this information is very important for making a decision. Scientists are tackling these problems. Kosylo, Smith, Conover, Chan, Zhang, Mei, and Cao proposed the prediction of jobhopping patterns where many profiles are incomplete based on novel AI technology, Sequential Optimization of Naive Bayesian (SONB) [18].

AI cannot work without data from experience. If current management practices are biased, discriminated, or overly hierarchical, the situation can only worsen. The authors note that machines need to be controlled to avoid autonomous decisions and implicit biases [12].

The desire to collect as much data as possible about employees for artificial intelligence systems can induce employers to violate privacy boundaries. There is a risk of disclosure as well as unintentional misuse of the data. For example, the system reports a high likelihood of a high-performing employee leaving the company and management may change their attitude towards this employee. In this regard, it is important to explain the decision made by the system: it must tell why it made it so that people can decide whether this decision is appropriate. Technology should help managers and HR professionals in organizations but not replace them [3]. A lot of difficulties remain in the event of a system failure: it will take a lot of time to analyze the current situation, understand which systems have failed, and also study what could lead to a similar situation.

Another limiting factor is that companies lack general knowledge about AI, including among C-level executives [8]. Due to the research results, we can see that companies in Russia also offer AI-based products for HR but they are not as diverse as in the world. According to a survey conducted by Rekadro among 188 respondents - representatives of HR services of Russian enterprises, the use of AI in practice is still small - $9 \%$ use artificial intelligence in 2020. However, on the whole, the dynamics are positive.

The main limitation of the study is the lack of quantitative information about the subject under study. Speaking about the prospects for the development of AI in HRM it should be noted that AI has significant potential. Perhaps it is worth paying more attention to constraints. Also, it is necessary to study in detail companies using AI in HR functions for future research. It is interesting to study possible changes in the HR manager profession and the role of HR in business.

\section{Conclusions}

Artificial intelligence provides many opportunities for modern companies: selection of employees when hiring, formation of a personnel reserve, identification of employees with high potential, selection for training and leadership development programs, etc. Most of the tools are easy to implement, take a little time, and the investment in them pays off very quickly. The potential for using AI is very large. With the development, increased reliability, and greater focus of AI-based systems on solving specific human resource management problems, we should see major improvements in employee productivity, efficiency, and well-being.

Despite the benefits of using AI, it creates challenges for companies, developers, government, and employees. The main task is to reskill the workforce to use AI, not to compete with it. The development of AI requires addressing ethical, legal, and regulatory issues that might otherwise hold back the development of AI [6]. In the context of changing employees according to generational theory, the development and application of AI can accelerate, which is a direction for further research. 


\section{References}

1. S.Y. Barykin, I.V. Kapustina, T.V. Kirillova, V.K. Yadykin, Y.A. Konnikov, J. Open Innov. Technol. Mark. Complex 6(4), 1-16 (2020) doi: 10.3390/joitmc6040124

2. IBM Corporation, Extending expertise (2017)

3. D.L. Stone, D.L. Deadrick, K.M. Lukaszewski, R. Johnson, Hum. Resour. Manag. Rev. 25(2), 216-231 (2015) doi: 10.1016/j.hrmr.2015.01.002

4. S. Evseeva, O. Evseeva, O. Kalinina, E3S Web Conf. 175 (2020) doi: 10.1051/e3sconf/202017513041.

5. A European approach to Artificial intelligence. Shaping Europe's digital future, https://digital-strategy.ec.europa.eu/en/policies/european-approach-artificialintelligence

6. Artificial intelligence the next digital frontier? (McKinsey Global Institute, 2017) https://www.mckinsey.com/ /media/mckinsey/industries/advanced electronics/our insights/how artificial intelligence can deliver real value to companies/mgi-artificialintelligence-discussion-paper.ashx

7. Global survey: The state of AI in 2020 (McKinsey) https://www.mckinsey.com/business-functions/mckinsey-analytics/our-insights/globalsurvey-the-state-of-ai-in-2020

8. E. Konnikov, O. Konnikova, V. Leventsov, IOP Conf. Ser. Mater. Sci. Eng. 497(1) (2019) doi: 10.1088/1757-899X/497/1/012043

9. G. Bhardwaj, S. V. Singh, V. Kumar, Proc. Int. Conf. Comput. Autom. Knowl. Manag. ICCAKM 2020, 47-51 (2020) doi: 10.1109/ICCAKM46823.2020.9051544

10. The social enterprise at work: Paradox as a path forward. 2020 Delloitte Global Human Capital Trends

https://www2.deloitte.com/content/dam/Deloitte/at/Documents/human-capital/at-hctrends-2020.pdf

11. E. Prem, Technol. Innov. Manag. Rev. 9(12), 5-15 (2019) doi: 10.22215/timreview/1287

12. A. Kaplan, M. Haenlein, Bus. Horiz. 62(1), 15-25 (2019) doi: 10.1016/j.bushor.2018.08.004

13. AI in HR: A Real Killer App - JOSH BERSIN, https://joshbersin.com/2018/06/ai-in-hra-real-killer-app/

14. O. Evseeva, O. Kalchenko, S. Evseeva, K. Plis, Atl. Highlights Comput. Sci., 148-154 (2019) doi: 10.2991/icdtli-19.2019.29

15. F.L. Oswald, T.S. Behrend, D.J. Putka, E. Sinar, Annu. Rev. Organ. Psychol. Organ. Behav. 7, 505-533 (2020) doi: 10.1146/annurev-orgpsych-032117-104553

16. C.B. Frey, M.A. Osborne, Technol. Forecast. Soc. Change 114, 254-280 (2017) doi: 10.1016/j.techfore.2016.08.019

17. M.M. Abdeldayem, S.H. Aldulaimi, Int. J. Sci. Technol. Res. 9(1), 3867-3871 (2020)

18. N. Kosylo et al., PICMET 2018 - Portl. Int. Conf. Manag. Eng. Technol. Manag. Technol. Entrep. Engine Econ. Growth, Proc., 10-14 (2018) doi: 10.23919/PICMET.2018.8481823 